\title{
Role of Hair Transplantation in Scarring Alopecia-To Do or Not to Do
}

\author{
Sukhbir Singh ${ }^{1}$ Kumaresan Muthuvel $^{2}$ \\ ${ }^{1}$ Department of Plastic Surgery, Resplendent the Cosmetic Studio, \\ New Delhi, India \\ 2 Department of Dermatology, Cutis Skin Clinic and Hair Transplant \\ Centre, Coimbatore, Tamil Nadu, India \\ Indian J Plast Surg 2021;54:501-506.
}

\begin{abstract}
Address for correspondence Dr. Sukhbir Singh, MS, DNB, Department of Plastic Surgery, Resplendent the Cosmetic Studio, R-9, Greater Kailash Part 1, New-Delhi-110048, India (e-mail: sukhi_1@yahoo.com).
\end{abstract}
Abstract
Keywords
- cicatricial alopecia (CA)
- hair transplant
- hair follicle
- frontal fibrosing alopecia (FFA)
- central centrifugal cicatricial alopecia (CCCA)

\begin{abstract}
Alopecia in the scalp region leads to psychosocial embarrassment for an individual. Alopecia could be due to several reasons, including genetic, hormonal, traumatic and infections. Cicatricial alopecias (CAs) are considered as trichological emergency, since their progression is rapid and always results in permanent hair loss. The pathogenesis, disease progression and prognosis of CA are poorly understood, and the treatment process is still evolving. An early diagnosis must be established, and aggressive treatment protocol should be followed in the management of scarring alopecia. This article presents various aspects of CA and determines whether hair transplant $(\mathrm{HT})$ should be done in this condition.
\end{abstract}

\section{Introduction}

Cicatricial alopecia (CA) or scarring alopecia is a group of uncommon inflammatory hair loss disorders characterized by permanent destruction of hair follicles. Clinically, there is a loss of visible follicular ostia over the scarring area, with or without epidermal atrophy; histologically, fibrous tracts replace the pilosebaceous structures. ${ }^{1-5}$ This leads to permanent hair loss in the scarred zone. It can involve any area on the scalp, including the facial regions. The available pharmacological treatments can prevent the progression of the disease to some extent. Hair transplant (HT) for CA is often explored (-Figs. 1-3) but many critical questions regarding the time of surgery, surgical technique, goal to achieve, how to decide the disease remission, and whether the surgical process might trigger the relapse of the disease remain unanswered. This review intends to summarize the available literature on HT in CA and come out with recommendations for the same.

\section{Classification}

The causes of CA are broadly classified as primary, secondary and hereditary or developmental defects. ${ }^{4-6}$ In primary cicatricial alopecia (PCA), there is irreversible hair loss from the affected site on the scalp, and the inflammatory cells target and destroy the stem cells in the bulge area of hair follicles. ${ }^{4}$ Based upon the histopathological picture, the CAs are divided mainly into lymphocyte-mediated primary cicatricial alopecia (LMPCA), neutrophil-mediated primary cicatricial alopecia (NMPCA), and mixed $\mathrm{CA}^{7}$ (-Table 1).

In secondary cicatricial alopecia (SCA), the hair follicles are secondarily damaged as a result of destructive processes published online

December 27, 2021
DOI https://doi.org/ $10.1055 / \mathrm{s}-0041-1739246$. ISSN 0970-0358.

\footnotetext{
(c) 2021. Association of Plastic Surgeons of India. All rights reserved. This is an open access article published by Thieme under the terms of the Creative Commons Attribution-NonDerivative-NonCommercial-License, permitting copying and reproduction so long as the original work is given appropriate credit. Contents may not be used for commercial purposes, or adapted, remixed, transformed or built upon. (https://creativecommons.org/ licenses/by-nc-nd/4.0/)

Thieme Medical and Scientific Publishers Pvt. Ltd., A-12, 2nd Floor, Sector 2, Noida-201301 UP, India
} 


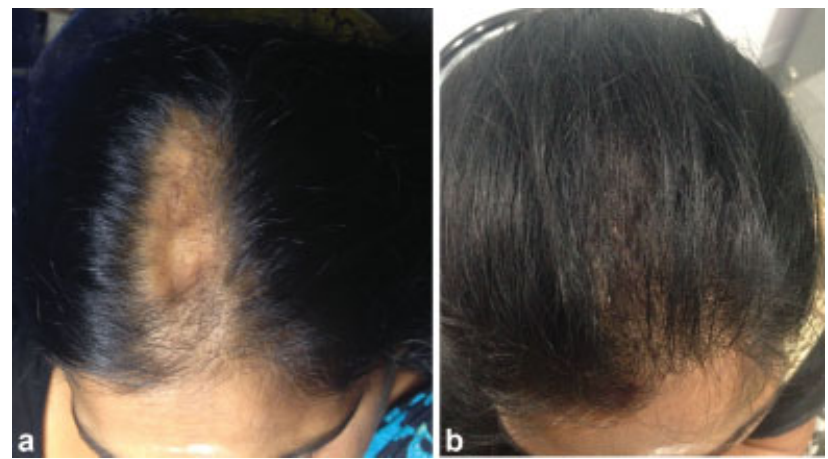

Fig. 1 (a) Preprocedure picture, (b) postprocedure picture.
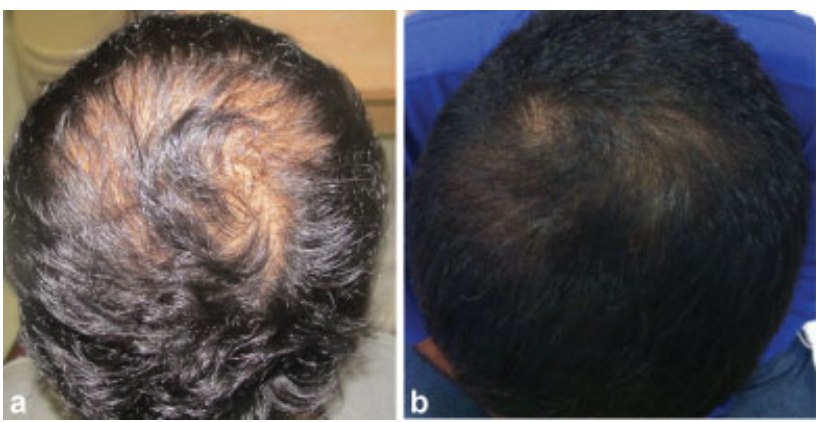

Fig. 2 (a) Preprocedure picture, (b) postprocedure picture.
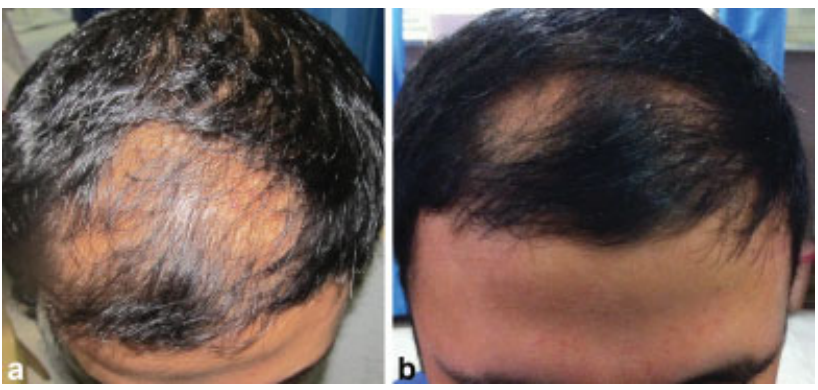

Fig. 3 (a) Preprocedure picture, (b) postprocedure picture.

within the skin. ${ }^{5}$ The causes of SCA are elaborated in - Table 1.

Another classification categorizes CA into "stable" and "unstable" type ${ }^{8}$. "Stable" CAs are secondary to isolated events that cause permanent scarring in a hair-bearing region. Whereas "unstable" cicatricial alopecias (UCAs) are secondary to disorders that tend to progress and recur intermittently over the course of time.

\section{Pathogenesis}

The pathogenesis of PCA revolves mainly around the destruction of slow-cycling, pluripotent hair follicle stem cells (HFSCs). ${ }^{9,10}$ These HFSCs are located in the "bulge" region of a hair follicle in the outer root sheath (i.e., at the site of attachment of the arrector pili muscle to the outer root
Table 1 Classification of Cicatricial alopecia

\begin{tabular}{|l|l|}
\hline Primary CA & Secondary CA \\
\hline Lymphocyte-associated: & Infections: \\
CCLE & Bacterial, viral, fungal \\
LPP & Immunological: \\
Classical type & Sarcoidosis, necrobiosis \\
Frontal fibrosing alopecia & lipoidica, morphea, graft \\
Graham-Little syndrome & versus host disease. \\
Classic pseudopelade (Brocq) & Neoplasms: \\
CCCA & Alopecia mucinosa, \\
Alopecia mucinosa & lymphoproliferative \\
Keratosis follicularis & Exogenous: \\
spinulosa decalvans & Burns and surgical and \\
Neutrophil-associated: & traumatic scars. \\
Folliculitis decalvans & Dermatoses: \\
Dissecting cellulitis/ & Psoriasis, immunobullous \\
folliculitis (perifolliculitis & disorders \\
abscendens et suffodiens) & Hamartomas: \\
Mixed inflammatory & Organoid nevus \\
infiltrate: & Miscellaneous \\
Folliculitis (acne) keloidalis & \\
Folliculitis (acne) necrotica & \\
Erosive pustular dermatosis & \\
Nonspecific & \\
\hline
\end{tabular}

Abbreviations: CA, cicatricial alopecia; CCCA, central centrifugal cicatricial alopecia; CCLE, chronic cutaneous lupus erythematosus; LPP, lichen planopilaris

sheath). The newer insights into the pathogenesis of PCA mainly involves the HFSCs' destruction theories, impairment of self-maintenance of HFSCs, alteration of lipid metabolism, neurogenic inflammation theory, and environment and genetic factors. ${ }^{4,11-30}$ Although various treatment modalities are presently based on immune-modulation, none is definite or completely curative.

In contrast, as the pathogenesis of SCA is already known (due to trauma, infection or others), various surgical modalities are present to give definite results in case of SCA. ${ }^{31-35}$ There is always a tell-tale sign in cases of secondary cases like burns in other areas, visible sutured, scarred zone, history of previous infection, or radiation therapy postcancer treatment.

\section{Clinical Evaluation in CA}

Primary CA presents a therapeutic challenge. During the initial phase of the disease, process symptoms and signs might mimic androgenetic alopecia (AGA). In the later stages of CA, the clinical picture looks similar in all the subtypes of PCA. A proper history, clinical examination, trichoscopic evaluation, and scalp biopsy helps in arriving at appropriate diagnosis. Majority of PCA occur in early adult life, with the exception of the two genetic conditions, keratosis follicularis spinulosa decalvans and Marie Unna hypotrichosis, which are seen in childhood. ${ }^{36}$ Frontal fibrosing alopecia (FFA) occurs in the postmenopausal age group. Central centrifugal cicatricial alopecia (CCCA), acne keloidalis, and dissecting cellulitis of scalp are seen in people of African origin. ${ }^{37}$ Patients with PCA usually present with associated symptoms 
of scalp scaling, pigmentation, itching, irritation, pustules, swelling on scalp, active hair loss in patches or a diffuse loss, hair loss in other regions of the body apart from the scalp, and pain on the scalp. These symptoms when present help the physician to think of PCA while evaluating a patient with hair loss. ${ }^{38}$ Location of the lesions provides the clue to the diagnosis of PCA. ${ }^{38}$ Vertex lesions are seen in CCCA and dissecting cellulitis of scalp. Frontal involvement is seen in FFA. Acne keloidalis is seen in the nape of the neck.

Chronic cutaneous lupus erythematosus (CCLE) presents with maximal clinical activity in the center. Perifollicular inflammation, follicular plugging, atrophy, and dyspigmentation are the characteristic findings. ${ }^{38}$ In lichen planopilaris (LPP), the clinical activity is seen in periphery of the lesions, with features of perifollicular erythematous and violaceous papules, spinous follicular hyperkeratosis and multifocal disease. ${ }^{38}$ Associated findings of oral cavity, skin and nails characteristic of lichen planus may be observed in cases of LPP. ${ }^{1}$ FFA presents with a band-like alopecia over the frontal region of the postmenopausal women. Involvement of eyebrow hair may be observed in FFA. ${ }^{38}$ Diagnosing the subtypes of long-standing PCA clinically poses a challenge, since atrophic scarring is seen in all the conditions of PCA, except folliculitis decalvans which present $s$ with hypertrophic scarring. ${ }^{38}$ Features suggestive of dissecting cellulitis of scalp are pustules, honey-colored crusts at the periphery of the lesions, and tufting of the hair. ${ }^{38}$ In dissecting cellulitis of scalp, the features observed are purulent discharge, boggy scalp, and sinus tracts. ${ }^{38}$

Diagnosis of CA is made by correlating clinical, trichoscopic, and scalp biopsy findings. Scalp biopsy should be done on the active margins of the CA lesions. ${ }^{39}$ Two biopsy specimens are recommended for vertical and horizontal sections to analyze the involvement of various structures of the hair follicle. Hair pull test performed at the margins of the CA lesions indicate the activity of the disease. A positive hair pull test denotes active disease.

\section{Management}

PCA throws up a diagnostic and therapeutic dilemma to the treating surgeon. The aim of treatment currently focuses on reducing symptoms and reducing or stopping the progression of the disease. The mainstay of treatment of LMPCA is immunosuppression and of NMPCA is antimicrobials or dapsone. ${ }^{40}$ In cases of PCA, surgical treatment can be planned only after complete disease stability, which is sometimes never achieved. Chloe et al in a systematic review of hair transplant in PCA found a $76 \%$ positive outcome rate. ${ }^{41}$ Eight out of 34 patients experienced negative results, including those with a diagnosis of LPP or FFA. FFA had the worst results.

In cases of secondary scarring alopecia, we have various options including hair transplantation, scalp reduction surgeries, tissue expansion and flap surgeries. ${ }^{8,42,43}$ There are various limitations in case of secondary alopecias due to the excessive scarring, which can occur in burns and postradiation patients. Umar described successful results in three cases of secondary alopecias using non-scalp donor sites. ${ }^{44}$

\section{Challenges Faced in Hair Transplant (HT) for CA due to the Following Factors:}

1. The question pertaining to when to opt for the surgical treatment remains unanswered. The common consensus is that a quiescent disease phase of 2 years is necessary before the surgical treatment. ${ }^{8}$ The difficulty in documenting the disease inactivity poses a further dilemma to the surgeon.

2. The vascularity of the scar tissue is unpredictable and varies in terms of interpatient and intrapatient variables.

3. Graft survival in scar tissue is unpredictable.

4. There is always a risk of disease relapse and loss of grafted follicular units (FUs).

5. In extensive scarring, donor insufficiency may limit the possibility of HT.

6. Multiple sessions are required for a good cosmetic outcome.

7. Possibility of future evolution of male pattern baldness (MPB)/female pattern hair loss (FPHL) should be considered in every CA undergoing HT around the areas treated.

8. The surgical procedure in itself might trigger the relapse of CA, which is reported in cases of LPP.

9. Wound healing characteristics vary widely and remain unpredictable.

10. Common complications encountered after HT are hypertrophic scars, graft rejection, infection, and corkscrew hair.

\section{Factors to Be Considered before Planning HT in CA}

\section{Donor-Recipient Area Mismatch}

Estimation of the donor-recipient area remains the most critical decision in choosing HT for CA. In the majority of the patients, the available donor FUs are insufficient to treat the existing as well as the probable future areas of CA. This is more so in those patients with CA involving the donor area. Body hair harvesting should be considered in all patients to optimize the donor reserves.

\section{Scar Texture}

In atrophic scar tissue, the recipient sites depth may be shallow and not be sufficient to accommodate the entire implanted FU due to lack of soft tissues; moreover, there is risk of insufficient perfusion to the implanted FU. In hypertrophic scars, the implanted follicles might not reach adequate depth to take up the blood supply from the subcutaneous tissue due to the thickness of scar tissue. A CA scar can have atrophic and hypertrophic components in the same lesion. History of hypertrophic scar/keloid over the previous surgical sites indicates a poor prognosis and requires careful planning.

\section{Location of the Scar}

Scars involving the hair line requires careful planning with a relatively high-density FU (25-35 FU/cm), since these regions have good adjacent vascularity. Shao et al used a 
follicular density of 20 to $48 \mathrm{FU} / \mathrm{cm}^{2}$ in his series. ${ }^{45} \mathrm{FU}$ placed on the normal-looking skin adjacent to the scars provides good camouflage. Vertical scars are difficult to conceal than the horizontal or oblique scar, since vertical scars need long hair to fall over the alopecia in order to provide cover.

\section{Scar Vascularity}

Grafts at the center of a large scar are distant from a good vascular supply, and ischemic injury may lead to necrosis and infection in addition to poor growth of the FU. Vascularity might not be uniform all over the scar tissue.

\section{Alopecia Reduction Versus HT}

HT is preferable to scar excision/alopecia reduction (AR) in patients with minimal scalp laxity, those with scalp where there are limitations in surgical undermining of the tissue due to lack of stretch of scalp intraoperatively, poor donor reserves (Hamilton Norwood scale 7), very young patients with evolving hair loss, and those with unrealistic expectations. ${ }^{46}$ Good candidates for AR are those 40 years and above with stable hair loss pattern (Hamilton-Norwood scale 4, 5, 6), good donor hair in the occipital region, smaller/solitary CA lesion, and those who accept hair loss on the incision lines. ${ }^{46}$ Scalp flaps can be considered in cases of CA, where local flaps can be utilized without having a secondary defect to restore. ${ }^{47}$

\section{Techniques to Be Modified to Improve the Cosmetic Outcome of HT in CA}

\section{Assessing Vascularity of the Scar Tissue}

Adrenaline usage should be avoided in the recipient site, as vascularity is already compromised due to scarring. To test the blood supply of a large area, it is recommended that a portion of the recipient area is anaesthetized with a $2 \%$ lidocaine solution without epinephrine. ${ }^{8} \mathrm{~A} 19-\mathrm{G}$ needle can be used to make several incisions on the recipient scar tissue. The evidence of bleeding during the incisions with the needle denotes the presence of adequate vascularity in the tissue. The absence of bleeding points indicates compromised blood supply, and the options of other surgical modalities should be explored. The vascularity of the scar tissue should be periodically assessed intraoperatively within the same scar tissue by evaluating the bleeding while making the recipient area incisions.

\section{FU Graft Size Selection}

Follicular family ([FF]-two closely placed FUs) are useful in creating an illusion of density in a scarred area. FF grafts provide the option of implanting a greater number of hair follicles within the same recipient site and reduce the need to make multiple incisions, minimizing the vascular damage. ${ }^{8}$

\section{Recipient Area Slit Making for Implantation of FU Grafts}

Recipient sites should be smaller in tissue with great laxity or very little recoil to prevent the popping of grafts, whereas sites need to be larger if the scar has limited laxity or significant recoil to prevent excessive manipulation of grafts during insertion. ${ }^{8}$ To minimize the vascular damage in the recipient area, it is recommended to make the recipient site incisions parallel to the direction of hair growth. ${ }^{8}$ While making the recipient site incisions parallel to the existing hair follicles, acute angle of incision leads to increased depth at the level of epidermis compared with less acute angle of incision in spite of using the same size needle or blade. In recipient sites requiring acute angle incisions (eyebrow, temporal regions, and the hairline in supra-auricular and preauricular regions), the diameter of the needle/size of the recipient slits should be reduced to prevent wider and deeper implantation slits over the scarred tissue. ${ }^{8}$ Atrophic scars are too shallow to accommodate and adequately perfuse the FU. Hence, the recipient incision has to be made deeper by creating an incision at a more acute angle. In hypertrophic scars, the recipient sites have to be made deeper than usual to improve the perfusion to the FU.

\section{Test Grafting and Density of FU in Recipient Area}

Patients with CA can be subjected to a test graft session and wait time of 6 months to assess the outcome before venturing into a full procedure. ${ }^{48}$ The density of FU per $\mathrm{cm}$ is the most difficult decision a surgeon needs to make while doing HT in CA. The standard recommendation is not to perform higher density FU grafting in CA. In CA regions with poor blood supply, FU densities of 15 to $20 \mathrm{FU} / \mathrm{cm}^{2}$ are considered optimal for better survival of the FU. In CA lesions with good blood supply, the density of FU can be 20 to $30 \mathrm{FU} / \mathrm{cm}^{2}$. To provide optimal density of hairs for a better coverage, the CA lesions can be grafted in a staged manner; a second surgery 9 to 12 months after first surgery is recommended to optimize the FU survival. Scalp tissue tends to be thicker in atrophic regions of $\mathrm{CA}$, and vascularity increases following first HT surgery. ${ }^{49}$

\section{Adjuvant Treatment}

Topical minoxidil (2-5\%) application in the recipient area for 2 weeks before and at least 5 weeks after surgery increases the local blood supply, prolongs anagen (hair growth) phase, and leads to subsequent improvement in graft survival. ${ }^{49}$

Pentoxifylline, $400 \mathrm{mg}$, thrice a day with meals, for 2 weeks prior to surgery has been advised to increase the oxygenation in the recipient area. ${ }^{50}$

Intralesional steroid injection in a stepwise manner into the scar tissue prior to HT has been tried with good outcome, in order to reduce the thickness of the scar and increase the pliability. ${ }^{49}$

Prior treatment of the scar tissue with fractional co2 lasers have been reported to be beneficial in improving the outcome of FU grafting in CA. The accelerated growth of transplanted hairs has been reported due to the promotion of neoangiogenesis and the inductions of various growth factors and cytokines during the wound healing process after laser-induced thermal damage. A hole-to-hole interval of $5 \mathrm{~mm}$ is assumed to be suitable for laser pretreatment of the recipient area in $\mathrm{CA}^{51}$ 
Platelet-rich plasma (PRP) has been tried with good success as an adjuvant in CA to improve the outcome of HT. PRP has been tried prior to the surgery, during the surgery on the recipient area sites, and postsurgery management. PRP influences wound healing and scar remodeling and may provide better FU survival. ${ }^{52}$

Additional treatments like topical steroids/dapsone/other immunosuppressants may need to be continued post-HT, especially in PCA, to prevent disease progression/relapse.

\section{Conclusion}

HT is a definite option for CA, more so in secondary CA as compared with primary $C A$. In primary $C A$, a waiting period of 2 to 5 years should be observed for complete disease stability before undertaking HT. A proper counselling should be given about the possibilities of negative results, especially in FFA and LPP, chances of disease relapse, or reactivation after HT. Emphasis on continuing the medical management should be provided even after HT in cases of primary CA.

\section{Financial Disclosures \\ None}

\section{Conflict of Interest}

None declared.

\section{References}

1 Whiting DA. Cicatricial alopecia: clinico-pathological findings and treatment. Clin Dermatol 2001;19(02):211-225

2 Tan E, Martinka M, Ball N, Shapiro J. Primary cicatricial alopecias: clinicopathology of 112 cases. J Am Acad Dermatol 2004;50(01): 25-32

3 Griffin LL, Michaelides C, Griffiths CE, Paus R, Harries MJ. Primary cicatricial alopecias: a U.K. survey. Br J Dermatol 2012;167(03): 694-697

4 Ohyama M. Primary cicatricial alopecia: recent advances in understanding and management. J Dermatol 2012;39(01): 18-26

5 Paus R, Olsen E, Messenger A. Hair growth disorders. In: Wolff K, Goldsmith LA, Katz SI, Gilchrest BA, Paller AS, Leffell DJ, eds. Fitzpatrick's Dermatology in General Medicine. 7th ed. New York: McGraw-Hill; 2008:753

6 Sinclair RD. Acquired cicatricial alopecia. In: Burns T, Breathnach SM, Cox N, Griffiths CE, eds. Rook's Textbook of Dermatology. 8th ed. Oxford: Wiley-Blackwill Publishing; 2010:66.38-66.52

7 Rongioletti F, Christana K. Cicatricial (scarring) alopecias: an overview of pathogenesis, classification, diagnosis, and treatment. Am J Clin Dermatol 2012;13(04):247-260

8 Unger W, Unger R, Wesley C. The surgical treatment of cicatricial alopecia. Dermatol Ther 2008;21(04):295-311

9 Sellheyer K, Bergfeld WF. Histopathologic evaluation of alopecias. Am J Dermatopathol 2006;28(03):236-259

10 Lavker RM, Sun TT, Oshima H, et al. Hair follicle stem cells. J Investig Dermatol Symp Proc 2003;8(01):28-38

11 Harries MJ, Paus R. The pathogenesis of primary cicatricial alopecias. Am J Pathol 2010;177(05):2152-2162

12 Cotsarelis G. Epithelial stem cells: a folliculocentric view. J Invest Dermatol 2006;126(07):1459-1468

13 Paus R, Cotsarelis G. The biology of hair follicles. N Engl J Med 1999;341(07):491-497
14 Pozdnyakova O, Mahalingam M. Involvement of the bulge region in primary scarring alopecia. J Cutan Pathol 2008;35(10): 922-925

15 Al-Refu K, Edward S, Ingham E, Goodfield M. Expression of hair follicle stem cells detected by cytokeratin 15 stain: implications for pathogenesis of the scarring process in cutaneous lupus erythematosus. Br J Dermatol 2009;160(06):1188-1196

16 Cotsarelis G, Millar SE. Towards a molecular understanding of hair loss and its treatment. Trends Mol Med 2001;7(07): 293-301

17 McElwee KJ. Etiology of cicatricial alopecias: a basic science point of view. Dermatol Ther 2008;21(04):212-220

18 Nijhof JG, Braun KM, Giangreco A, et al. The cell-surface marker MTS24 identifies a novel population of follicular keratinocytes with characteristics of progenitor cells. Development 2006;133 (15):3027-3037

19 Jensen KB, Collins CA, Nascimento E, et al. Lrig1 expression defines a distinct multipotent stem cell population in mammalian epidermis. Cell Stem Cell 2009;4(05):427-439

20 Amoh Y, Li L, Katsuoka K, Penman S, Hoffman RM. Multipotent nestin-positive, keratin-negative hair-follicle bulge stem cells can form neurons. Proc Natl Acad Sci U S A 2005;102(15): 5530-5534

21 Jaks V, Barker N, Kasper M, et al. Lgr5 marks cycling, yet long-lived, hair follicle stem cells. Nat Genet 2008;40(11):1291-1299

22 Snippert HJ, Haegebarth A, Kasper M, et al. Lgr6 marks stem cells in the hair follicle that generate all cell lineages of the skin. Science 2010;327(5971):1385-1389

23 Harries MJ, Meyer KC, Chaudhry IH, Griffiths CE, Paus R. Does collapse of immune privilege in the hair-follicle bulge play a role in the pathogenesis of primary cicatricial alopecia? Clin Exp Dermatol 2010;35(06):637-644

24 Baima B, Sticherling M. Apoptosis in different cutaneous manifestations of lupus erythematosus. Br J Dermatol 2001;144(05): 958-966

25 Pablos JL, Santiago B, Galindo M, Carreira PE, Ballestin C, GomezReino JJ. Keratinocyte apoptosis and p53 expression in cutaneous lupus and dermatomyositis. J Pathol 1999;188(01):63-68

26 Nakajima M, Nakajima A, Kayagaki N, Honda M, Yagita H, Okumura K. Expression of Fas ligand and its receptor in cutaneous lupus: implication in tissue injury. Clin Immunol Immunopathol 1997;83(03):223-229

27 Zheng Y, Eilertsen KJ, Ge L, et al. Scd1 is expressed in sebaceous glands and is disrupted in the asebia mouse. Nat Genet 1999;23 (03):268-270

28 Sundberg JP, Boggess D, Sundberg BA, et al. Asebia-2J (Scd1 (ab2J)): a new allele and a model for scarring alopecia. Am J Pathol 2000;156(06):2067-2075

29 Stenn KS. Insights from the asebia mouse: a molecular sebaceous gland defect leading to cicatricial alopecia. J Cutan Pathol 2001;28 (09):445-447

30 Karnik P, Tekeste Z, McCormick TS, et al. Hair follicle stem cellspecific PPARgamma deletion causes scarring alopecia. J Invest Dermatol 2009;129(05):1243-1257

31 Gho CG, Neumann HA. Improved hair restoration method for burns. Burns 2011;37(03):427-433

32 Orentreich N. Autografts in alopecias and other selected dermatological conditions. Ann N Y Acad Sci 1959;83:463-479

33 Limmer BL. Elliptical donor stereoscopically assisted micrografting as an approach to further refinement in hair transplantation. J Dermatol Surg Oncol 1994;20(12):789-793

34 Rassman WR, Carson S. Micrografting in extensive quantities. The ideal hair restoration procedure. Dermatol Surg 1995;21(04): 306-311

35 Bernstein RM, Rassman WR. Follicular transplantation. Patient evaluation and surgical planning. Dermatol Surg 1997;23(09): 771-784, discussion 801-805 
36 Sperling LC, Sau P. The follicular degeneration syndrome in black patients. 'Hot comb alopecia' revisited and revised. Arch Dermatol 1992;128(01):68-74

37 Filbrandt R, Rufaut N, Jones L, Sinclair R. Primary cicatricial alopecia: diagnosis and treatment. CMAJ 2013;185(18):1579-1585

38 Shapiro J. Cicatricial alopecias. Dermatol Ther 2008;21(04):211

39 Harries M, Trueb RM, Tosti A, et al. How not to get scar(r) ed: pointers to the correct diagnosis in patients with suspected primary cicatricial alopecia. Br J Dermatol 2009; 160:482-501

40 Harries MJ, Sinclair RD, Macdonald-Hull S, Whiting DA, Griffiths CE, Paus R. Management of primary cicatricial alopecias: options for treatment. Br J Dermatol 2008;159(01):1-22

41 Ekelem C, Pham C, Atanaskova Mesinkovska N. A systematic review of the outcome of hair transplantation in primary scarring alopecia. Skin Appendage Disord 2019;5(02):65-71

42 Yang CC. Calvitron automated hair transplant system in alopecia treatment: a case report. Kaohsiung J Med Sci 2003;19(09):470-475

43 Shiell RC. Modern hair restoration surgery. Clin Dermatol 2001;19 (02):179-187

44 Umar S. Hair transplantation in patients with inadequate head donor supply using non-head hair: report of 3 cases. Ann Plast Surg 2011;67(04):332-5
45 Shao H, Hang H, Yunyun J, et al. Follicular unit transplantation for the treatment of secondary cicatricial alopecia. Plast Surg (Oakv) 2014;22(04):249-253

46 Sattur SS. A review of surgical methods (excluding hair transplantation) and their role in hair loss management today. J Cutan Aesthet Surg 2011;4(02):89-97

47 Unger MG, Unger WP. Management of alopecia of the scalp by a combination of excisions and transplantations. J Dermatol Surg Oncol 1978;4(09):670-672

48 Cooley J. Complications of hair transplantation. In: Unger WP, Shapiro R, eds. Hair Transplantation, 4th ed. New York: Marcel Dekker; 2004:573

49 Madura C, Vinay N, Kusuma MR, Harsha S, Chandrashekar BS. The assessment of hair transplantation outcomes in cicatricial alopecia. Int J Trichology 2020;12(04):164-167

50 Kutlubay Z, Kucuktas M, Engin B. Hair transplantation in the cicatricial alopecias. Hair Ther Transplant 2013;3:1-4

51 Kwon OS, Kim MH, Park SH, Chung JH, Eun HC, Oh JK. Staged hair transplantation in cicatricial alopecia after carbon dioxide laserassisted scar tissue remodeling. Arch Dermatol 2007;143(04): 457-460

52 Kumar AR, Ishii LE. Hair transplantation for scarring alopecia. Facial Plast Surg Clin North Am 2020;28(02):177-179 